\title{
Origin of Work Function Modification by Ionic and Amine-Based Interface Layers
}

\author{
Stephan van Reenen, Sandra Kouijzer, Rene A. J. Janssen, \\ Martijn M. Wienk and Martijn Kemerink
}

\section{Linköping University Post Print}

\section{Tweet}

N.B.: When citing this work, cite the original article.

Original Publication:

Stephan van Reenen, Sandra Kouijzer, Rene A. J. Janssen, Martijn M. Wienk and Martijn Kemerink, Origin of Work Function Modification by Ionic and Amine-Based Interface Layers, 2014, ADVANCED MATERIALS INTERFACES, (1), 8, 1400189.

http://dx.doi.org/10.1002/admi.201400189

Copyright: Wiley http://eu.wiley.com/WileyCDA/

Postprint available at: Linköping University Electronic Press http://urn.kb.se/resolve?urn=urn:nbn:se:liu:diva-114448 


\title{
WILEY-VCH
}

DOI: 10.1002/ ((please add manuscript number))

Article type: Full Paper

\section{Origin of work function modification by ionic and amine-based interface layers}

\author{
Stephan van Reenen, Sandra Kouijzer, René A. J. Janssen, Martijn M. Wienk, and Martijn
} Kemerink*

S. van Reenen, Prof. R. A. J. Janssen, Prof. M. Kemerink

Department of Applied Physics, Eindhoven University of Technology, P.O. Box 513, 5600

MB Eindhoven, The Netherlands

E-mail: martijn.kemerink@liu.se

Prof. M. Kemerink

Department of Physics, Chemistry and Biology (IFM), Linköping University, SE-58 183, Linköping, Sweden

S. Kouijzer, Prof. R. A. J. Janssen, Dr. M. M. Wienk

Department of Chemical Engineering, Eindhoven University of Technology, P.O. Box 513, 5600 MB Eindhoven, The Netherlands

Keywords: Organic electronics, Dipole formation, Photovoltaic devices, Kelvin probe, work function

Work function modification by polyelectrolytes and tertiary aliphatic amines is found to be due to formation of a net dipole at the electrode interface, induced by interaction with its own image dipole in the electrode. In polyelectrolytes differences in size and side groups between the moving ions lead to differences in approach distance towards the surface. These differences determine magnitude and direction of the resulting dipole. In tertiary aliphatic amines the lone pairs of electrons are anticipated to shift towards their image when close to the interface rather than the nitrogen nuclei, which are sterically hindered by the alkyl side chains. Data supporting this model is from scanning Kelvin probe microscopy, used to determine the work function modification by thin layers of such materials on different substrates. Both reductions and increases in work function by different materials were found to follow a general mechanism. Work function modification is found to only take place when the work function modification layer (WML) is deposited on conductors or semiconductors. On insulators no effect is observed. Additionally, the work function modification is 


\section{WILEY-VCH}

independent of the WML thickness or the substrate work function in the range of 3 to $5 \mathrm{eV}$. Based on these results charge transfer, doping, and spontaneous dipole orientation are excluded as possible mechanisms. This understanding of the work function modification by polyelectrolytes and amines facilitates design of new air-stable and solution-processable WMLs for organic electronics.

\section{Introduction}

The remarkable efficiency leap from less than $5 \%$ to over $10 \%$ in the past 7 years makes it plausible that organic solar cells can evolve into a successful thin film photovoltaic technology. To this end, further improvements in the sunlight-to-electricity conversion efficiency are still needed. To do so, not only the optoelectronic, active layer should be considered, but also the connection between this layer and the electrodes. This connection is facilitated by so-called work function modification layers (WMLs) that serve to align the transport levels in the organic semiconductor and the conductor by modification of the work function. Materials that are often used are poly(3,4-ethylenedioxythiophene) poly(styrenesulfonate) (PEDOT:PSS) for improving hole collection and injection in combination with an indium tin oxide (ITO) electrode and LiF for the electron contact in combination with an $\mathrm{Al}$ metal electrode. A new generation of WMLs first introduced by Cao et al. is based on polyelectrolytes or tertiary aliphatic amines, such as poly[(9,9-bis $\left(3^{\prime}-(\mathrm{N}, \mathrm{N}-\right.$ dimethylamino)propyl)-2,7-fluorene)-alt-2,7-(9,9-dioctylfluorene) $\quad$ (PFN) and the corresponding ethyl ammonium bromide. ${ }^{[1]}$ Although these materials improve carrier injection or extraction in different organic diode devices, ${ }^{[1-7]}$ the mechanism behind this improvement is not completely clear. This ambiguity hinders design, selection and optimization of new WMLs.

Mechanisms that are generally considered to result in electric fields and concomitant work function shifts at metal-organic interfaces are doping, charge transfer, and dipole 


\section{WILEY-VCH}

formation. ${ }^{[8-10]}$ Regarding the work function modification of amine side-groups, Lindell et al. ${ }^{[11]}$ found by photoelectron spectroscopy and density functional calculations that the electron donor para-phenylenediamine chemisorbs onto atomically clean $\mathrm{Ni}$ in vacuum. ${ }^{[12]}$ Due to partial electron transfer from the amine unit, a work function reduction of $1.55 \mathrm{eV}$ is achieved, resulting in a less deep Fermi level. This explanation is not likely to hold for the technologically more relevant procedure on which we shall focus, being the deposition of WMLs from solution on a metal at atmospheric pressure: the metal is not atomically clean, which likely inhibits chemisorption. In other work by Zhou et al. ${ }^{[13]}$ it is shown that the work function reduction, at atmospheric pressure, by the amine groups in poly(ethylenimine ethoxylated) (PEIE), is generally the same on different conductors. They attributed the effect both to i) orientation of the internal molecular dipole, induced by the lone pair of the nitrogen atom, and ii) an interfacial dipole due to partial charge transfer from the amine group after the physisorption on the conductor. Important for determining the origin of the effect is also the thickness dependence of the amine-containing layer. ${ }^{[13-17]}$ In this respect different observations have been made. Xia et al. ${ }^{[15]}$ reported an optimum of the work function modification at $8 \mathrm{~nm}$ thickness and above, while Zhou et al. ${ }^{[13]}$ and Zhang et al. ${ }^{[16]}$ did not observe any thickness dependence. Kim et al. ${ }^{[17]}$ reported a thickness dependence where thicker films resulted in a reduced work function modification.

Next to PFN, also cationic polyelectrolytes can be used to lower the work function of the electrode. ${ }^{[1,5-6]}$ A recent study on conjugated polymer zwitterions revealed a similar lowering in work function, attributed to electrostatic realignment of the dipolar zwitterionic side-chains near the metal interface. ${ }^{[18]}$ The formation of interfacial dipoles and electric fieldinduced reorientation of molecules has also been reported. ${ }^{[19]}$ Other work has shown that in the specific case that cationic polyelectrolytes are brought into contact with fullerenes, doping of fullerenes is observed. ${ }^{[20]}$ This however does not explain the work function modification of conductors in absence of fullerenes. ${ }^{[6]}$ 


\section{WILEY-VCH}

The anionic polyelectrolyte Nafion, which is also an acid, is found to give the opposite effect compared to cationic polyelectrolytes. A thin film of Nafion on ITO is found to increase the work function by $0.5 \mathrm{eV},{ }^{[21-23]}$ which indicates that Nafion can modify the metal work function. Another anionic polyelectrolyte is poly(4-styrenesulfonic acid) (H-PSS), a component of PEDOT:PSS formulations. In literature evidence exists that a thin layer of $\mathrm{H}$ PSS forms on top of PEDOT:PSS. ${ }^{[24]}$ Processing of PEDOT:PSS from aqueous dispersions with different amounts of added sorbitol was found to lead to a reduction in the work function, which has been interpreted as being the result of removal of the H-PSS top layer. ${ }^{[25]}$

In this work we present results which indicate that work function modification by anionic or cationic (poly)electrolytes and by tertiary aliphatic amines originates from a single mechanism: the formation of a dipole at the interface. This dipole formation is driven by the energetic stabilization by its image charges in the contacting (semi-)conductor. On metals coated with a $\sim 10 \mathrm{~nm}$ thin insulator no dipole orientation or formation is observed when an additional WML is applied: the work function is not modified. Equal work function modification is observed by scanning Kelvin probe microscopy for different thicknesses of PFN or H-PSS deposited on conductors with different work functions, ranging from Au to Ca. This excludes charge transfer or doping as a possible origin of the work function modification. The net direction of the dipole is furthermore determined by the ability of the positive and negative part of the dipole to get in the proximity of the (semi-)conductor. The ability to do so is found to depend on the geometric properties of the positive and negative part. This behavior is confirmed by series of tetrabutylammonium halides and H-PSS, Li-PSS, and Na-PSS. The magnitude of the net dipole can be related to both the ability to orient as well as the density of dipoles at the interface.

\section{Results and Discussion}




\section{WILEY-VCH}

To study the mechanism behind the work function modification by several types of WMLs, samples as depicted in Figure 1 were used. The parameter of interest is the work function of a conducting, semiconducting, or insulating layer with and without coverage of the WML. Insulators and intrinsic, i.e. undoped, organic semiconductors, however, do not have a welldefined work function as the Fermi level resides in the bandgap. Therefore thin insulating or semiconducting layers were deposited on top of a metal electrode as illustrated in Figure 1b. The electrostatic potential at the surface is measured by scanning Kelvin probe microscopy (SKPM) with respect to an Au-coated tip. The structural formulae of the materials used as WML or semiconducting/insulating substrate are shown in Figure 2.

\subsection{Work function reduction by PFN}

The effect of the WML PFN on substrates of conducting (also highly doped semiconductors like p-type doped $\mathrm{Si}$, p-type doped PEDOT:PSS and n-type doped $\mathrm{V}_{2} \mathrm{O}_{5},{ }^{[26]}$ prepared following the procedure reported in Ref. $\left[{ }^{27}\right]$ ), semiconducting (intrinsic), or insulating nature was measured as shown in Figure 3 and Table 1. The qualitative criterion for the classification of these substrate materials is based on the ability to provide charges, which is proportional to the dielectric response of the substrate material to the WML. Conductors have a significant amount of mobile charges, which is why we share highly doped semiconductors in this paper under the same denominator. Intrinsic semiconductors contain only few charges which are however mobile. Insulators do not contain mobile charges. In Figure 3, the horizontal axis represents the work function of each sample without coverage of the WML: the work function of the substrate. The vertical axis represents the work function shift of the sample after coverage with the WML. A negative work function modification corresponds to a reduction of the work function, i.e. improved electron injection from the substrate.

When PFN was used as WML the work function of the substrate is either reduced or constant as shown in Figure 3. In case the substrate is a conductor (black dots), an average 


\section{WILEY-VCH}

work function reduction of $0.7 \mathrm{eV}$ (gray line) is obtained. This value is independent of the substrate work function in the range measured here: from PEDOT with $\Phi \approx 5 \mathrm{eV}$ to Ca with $\Phi \approx 3 \mathrm{eV}$. Regarding $\mathrm{Ca}$ and $\mathrm{Al}, \mathrm{PFN}$ was spin coated from dry toluene rather than from methanol to prevent reaction of the solvent with the metal. The solvent from which PFN was deposited, either methanol with acetic acid, or toluene, did not affect the work function change by PFN for other metallic substrates. In case PFN is deposited on top of an organic semiconductor (blue squares), the work function is reduced as well, albeit slightly less compared to conductors: approximately $0.4 \mathrm{eV}$ (blue line). The work function modification by PFN was furthermore found to not depend on the thickness of the intrinsic, organic semiconductor, as verified for SY-PPV films with varying thickness between $7 \pm 3$ and $180 \pm 10$ nm. On insulators (red triangles) PFN did not result in any significant change in the work function (red line).

Another experiment to resolve the origin of the work function modification of conductors by PFN is the determination of the layer thickness dependence of the work function change. We deposited PFN with varying layer thickness on Au and on a thin film of SY-PPV on PEDOT:PSS and studied the thickness dependence of the work function by SKPM. Different layer thicknesses were obtained by spin coating solutions with different concentrations at a series of spin speeds ranging from 1000 to 4000 RPM. The work function modification of Au or SY-PPV by PFN was found to be constant for all these layers as shown in Figure 4: $0.7 \pm 0.1 \mathrm{eV}$ and $0.22 \pm 0.1 \mathrm{eV}$, respectively. It was verified that spin coating with solely the solvent did not modify the Au or PEDOT/SY-PPV work function.

In literature qualitatively different results have been reported regarding the dependence of the work function modification on the thickness of interlayers like PFN. ${ }^{[13-17,}$ ${ }^{28]}$ Increasing, ${ }^{[15]}$, constant, ${ }^{[13,16]}$ and decreasing ${ }^{[17]}$ work function shifts have all been reported. Part of these works comprises the characterization of complete devices, which complicates the interpretation as charge transport is involved as well. ${ }^{[14,16]}$ Use of thicker layers of insulating 


\section{WILEY-VCH}

materials will reduce the performance due to additional series resistance. Characterization of the open-circuit voltage circumvents this issue, although also the open-circuit voltage depends on many factors. Characterization of WMLs by inherently current-less techniques like $\mathrm{SKPM}^{[13,15,28]}$ excludes these problems, but still one must ensure that the substrate is completely covered. Incomplete coverage will result in intermediate results.

\subsection{Considered mechanisms}

To determine the nature of the work function reduction by PFN several models proposed in literature were considered first. ${ }^{[13,15,20]}$ For each model we inspected its ability to explain the experimental findings reported here and in literature: the work function modification is i) dependent on the nature of the substrate: metallic, (organic) semiconducting or insulating; ii) independent on the work function of the substrate in the range $3.0-5.0 \mathrm{eV}$; iii) independent of the PFN layer thickness; iv) possible in absence of any semiconductor which can be electrochemically doped. ${ }^{[13]}$

Four models that can describe the work function reduction by the WML PFN are schematically shown in Figure 5: formation of an electric double layer (EDL) after doping of the WML (Figure 5a), charge transfer to or from the conductor (Figure 5b), spontaneous dipole orientation in the WML (Figure 5c) and the spontaneous dipole orientation at the interface (Figure 5d). A detailed discussion follows in the next paragraphs about these mechanisms and their ability to describe the observations from Figure 3 and 4. Each of these mechanisms is found to have difficulties in explaining the complete series of findings described above. Therefore an alternative model is presented later on.

The first model (Figure 5a) describes electric double layer formation between a conductor and a doped material. In organics, doping can be achieved electrochemically by ions stabilizing electronic charges. Such behavior is e.g. the basis of light-emitting electrochemical cells. ${ }^{[29-31]}$ In the polyelectrolytes studied here, mobile ions are present that 


\section{WILEY-VCH}

can be used for electrochemical doping. Electrochemical doping is, however, not evident in the semiconductor PFN. Oxidation of tertiary aliphatic amines has nonetheless been reported and leads to the formation of a cationic compound. ${ }^{[32]}$ Such cationic compounds can stabilize electrons on nearby semiconductors, i.e. doping. The EDL that is formed in case doping is indeed present or takes place would result in a bias-dependent depletion of carriers in the doped semiconductor at the interface. The depletion width would range between 6 and $2 \mathrm{~nm}$ for (very high) doping densities of $10^{25}-10^{26} \mathrm{~m}^{-3}$, respectively, in case a change in built-in potential of $1 \mathrm{~V}$ is assumed. In our measurements no thickness dependence of work function modification by PFN is observed, which indicates that such a depletion layer is absent. Also the fact that PEIE, ${ }^{[13]}$ an insulating tertiary aliphatic amine, can change the work function without any form of doping indicates that the work function change studied in this paper is not due to doping and the resulting EDL formation. This however does not exclude that doping takes place in devices that contain polyelectrolytes. ${ }^{[20]}$ The relatively low ionic mobility can result in slow transient behavior during device operation. ${ }^{[5]}$

Modification of the work function can also occur due to dipole formation induced by integer or partial charge transfer between the substrate and the WML (Figure 5b). Charge transfer can take place in a large variety of circumstances as reviewed by Braun et al. in Ref. $\left.{ }^{8}\right]$. These can be divided in two classes: charge transfer after physisorption or chemisorption. The latter can occur in case the metal surface of the substrate is atomically clean, i.e. at pressures as low as $10^{-9}$ mbar. In contrast, the deposition of PFN reported here took place at atmospheric pressure. Nevertheless, in case a clean surface of the substrate is considered, specific interaction between the WML and the substrate can then take place. Specific interactions are, however, not likely to explain the work function modification by PFN on conductors as it is shown to be a general effect for multiple, different substrate materials in Figure 3. In case of physisorption, charge transfer can still take place. Charge transfer is driven by filling empty, lower lying energy states in either the substrate or the WML itself. 


\section{WILEY-VCH}

The cost of charge transfer is the electrostatic energy due to the formation of a dipole. In case of PFN on a metal the sign of the work function change could be explained by electron transfer into the metal. Figure 3 shows however that PFN reduces the work function of low work function metals like $\mathrm{Ca}$ and $\mathrm{Al}$ as much as e.g. Au. This implies that the electrondonating energy level in PFN would have to lie above the Fermi level of $\mathrm{Ca}$ and Al. Then PFN would not be air-stable, which it in fact is. Charge transfer therefore cannot explain the general behavior of PFN on conductors shown in Figure 3. Significant work function modification by charge transfer already occurs after deposition of a monolayers on e.g. a metallic substrate, ${ }^{[33]}$ which is in agreement with the absence of a thickness dependence of the work function modification by PFN.

A third mechanism to obtain a work function modification is the spontaneous orientation of dipoles in the WML deposited on top of the substrate. This spontaneous orientation of dipoles may either be throughout the complete layer (Figure 5c) or only at the interface (Figure 5d). In case the complete layer is polarized, a linear dependence of work function on layer thickness is expected as observed in Ref. [ $\left.{ }^{15}\right]$. Similar as in Ref. $\left[{ }^{16}\right]$ we find no thickness dependence as illustrated by Figure 4. Another argument against this model lies in the fact that we observe a work function reduction of organic semiconductors by PFN as well. This means that the positive end of the dipole in the WML is directed away from the organic semiconductor in absence of a top contact. This is opposite to devices reported in literature $^{[1]}$ and reproduced by us (Figure $\mathrm{S} 1$ in the supplementary information) where PFN deposition on the semiconductor is followed by deposition of Al. In these devices the dipole is directed towards the organic semiconductor as electron injection from $\mathrm{Al}$ is improved. This means that during or after deposition of $\mathrm{Al}$ the dipoles in the WML would have to be rotated by 180 degrees. Electric field-induced reorientation of polyelectrolytes has been reported. ${ }^{[19]}$ Here, however, no external field was applied. 


\section{WILEY-VCH}

In case the dipole orientation occurs only at the interface of the WML, an abrupt change in work function is expected as a function of the layer thickness. This is in line with our experimental findings. Still this mechanism has difficulties in explaining the inversion in dipole orientation between metal/organic semiconductor/PFN and metal/organic semiconductor/PFN/metal, as discussed in the previous paragraph. Before deposition of the top metal layer, the PFN molecules are randomly oriented. During evaporation, reorientation of those molecules should then occur to achieve a significant work function reduction. This process seems unlikely.

\subsection{Proposed mechanism}

Based on our experimental findings in addition to the literature cited in this article, we suggest the following mechanism for work function modification by PFN. Consider a material with positively and negatively charged constituents contacted by a conductor as shown in Figure 6. These charges would result in an electric field within the conductor. The material of the conductor, however, polarizes and by that produces an electric field to oppose this external electric field. ${ }^{34}$ The collective polarization of the medium can be regarded and described by the formation of image charges. The ability of materials to polarize and form such image charges depends on the static dielectric constant, which is large for metals or highly-doped semiconductors. Image charges of the positive and negative constituents are thus present in the conductor. ${ }^{[35]}$ As the image charges are oppositely charged, each image charge exerts an attractive force on its respective, real charge. Consequently, both charged constituents want to lie as close as possible near the conductor. The final position with respect to the conductor, however, depends on the geometrical size and the ability of the charged constituents to move. A large difference in size and ability to move of the charged constituents then leads to a difference in final position relative to the electrode. In Figure 6 this is illustrated in case both constituent parts have a) an equal and b) an unequal ability to move. The result of this 


\section{WILEY-VCH}

difference (Figure 6b) is that a dipole is created in the WML at the interface. This polarization follows a trade-off between the energy gain by approaching the metal surface and the energy loss of a net polarization. Note that the dipole of the image charges is in the same direction as the dipole of the real charges, enhancing the total dipole. This is due to the inverted sign of the charge of the image. In addition, the image dipole can enhance the effective polarizability of molecules at the interfaces of conductors. ${ }^{[36-37]}$ Recently, similar behavior as described in this paragraph was found in a zwitterionic polyelectrolyte ${ }^{[18]}$ and other polyelectrolytes ${ }^{[19]}$.

To understand the driving force of the work function modification, attraction by image charges in the substrate, the electrostatics of the system, the substrate and the WML should be taken into account. ${ }^{[38]}$ When the WML is physisorbed on an insulator, the only electrostatic interaction is that between the charged constituents of the molecules of the WML itself. The small difference between the relative dielectric constants of the WML and the insulator results only in minor image force interaction. In this case a net polarization would only cost energy and therefore does not occur: no work function modification takes place as shown in Figure 7c. In case the WML is physisorbed ${ }^{[13]}$ on a conductor an additional electrostatic interaction arises due to the formation of image charges. As discussed above, the interaction with these image charge makes it energetically favorable for (parts of) the physisorbed molecules to move closer to the conductor (Figure 7a). This is a very short range interaction, hence the virtual absence of a layer thickness dependence. Furthermore, for this effect to take place no charge transfer from the WML to the conductor or vice versa needs to take place. Consequently, the effect is independent of the work function of the conductor. For WMLs on semiconductors, the contrast in relative dielectric constant is similar as for insulators, i.e. small. However, semiconductors can hold compensation charges which can still stabilize a dipole. Hence energetically stable interface dipoles can be formed for WMLs physisorbed on semiconductors as shown in Figure $7 b$. 


\section{WILEY-VCH}

Our suggested mechanism to explain the experimental findings for PFN used as WML is based on the following facts. The work function reduction is independent of the work function of the layer below excludes that exchange of electrons with the conductor is of importance. The layer thickness independence of the work function modification additionally indicates that the effect occurs close to the interface as expected (Figure 7). No work function shift is observed in case an insulating layer is inserted between a conductor and the PFN. So, despite the absence of electron exchange between electrode and WML, a (semi)conducting surface is needed to enable work function modification. This is in line with the proposed mechanism that image charges in the (semi)conductor contacting the WML drive the polarization at the interface.

The relevant, charged constituents in PFN are the nitrogen atom and its lone pair in a $s p^{3}$ orbital. The relatively small $s$ character of the orbital results in a relatively large separation from the nucleus. The typical basicity of amines furthermore indicates that the lone pair of electrons is not delocalized over the molecule. ${ }^{[39]}$ Therefore this lone pair can in principle easily be attracted towards the interface by its image charge in the substrate.

\subsection{Work function increase by H-PSS and Nafion}

The proposed mechanism for work function modification discussed in the previous paragraph is based on the basic ingredients of having a (semi)conductive surface covered by molecules with charged constituents that differ in size and ability to move. Next to PFN, many other materials may possess these properties. Especially, polyelectrolytes can be expected to show similar behavior as illustrated in Figure 6 and 7. In literature quaternized ammonium derivatives of PFN have already been shown to improve electron injection similarly as PFN. ${ }^{[1,}$ ${ }^{6]}$ To determine whether other polyelectrolytes show similar behavior as PFN in work function modification of conducting, semiconducting, and insulating substrates, similar measurements 


\section{WILEY-VCH}

were performed with H-PSS and Nafion as WML. The results are shown in Figure 8 and Tables 2 and 3.

In case H-PSS or Nafion are used as WML the work function is observed to be increased or to remain constant. Similar to PFN, the strongest work function changes are observed on conductors (black dots or pentagons for H-PSS and Nafion respectively in Figure 8) and are within experimental uncertainty independent of the work function of the conductor itself. On organic semiconductors (blue squares or diamonds for H-PSS and Nafion respectively) only a small increase in work function is observed, whereas on insulators (red triangles for Nafion) a change in work function is absent. On semiconductors, the anionic polyelectrolytes (blue symbols in Figure 8) seem to result in a smaller work function modification as compared to PFN (blue symbols in Figure 3). This difference may be explained by the lesser ability of the used p-type semiconductors to provide an electron as compensation charge. In more detail, when a charged species sits close to an interface with an (intrinsic) semiconductor that is in contact with an electrode, electronic charges can be injected in the semiconductor and subsequently be transported to the interface, electrostatically compensating/screening the charged species at the interface. The end result is a dipole across the interface, like for the image charge mechanism which applies to materials with relatively high carrier densities like metals and highly-doped semiconductors. It is known from literature that p-type semiconductors are not good at providing compensating electrons and vice versa. ${ }^{[40-43]}$ As the deposition of a layer of H-PSS or Nafion occurs from an acidic solution, it was checked whether spin coating instead with a $1 \mathrm{M} \mathrm{H}_{2} \mathrm{SO}_{4}$ solution on $\mathrm{Au}$ gave similar results. This step actually results in washing the electrode with an acidic solution. No work function increase of the Au (star in Figure 8) was observed.

The results regarding PFN, H-PSS and Nafion shown in Figure 3 and 8 show that despite using different materials, the work function modification behaves the same, dependent on the type of substrate used. Different is however the sign of the work function modification 


\section{WILEY-VCH}

as PFN reduces the substrate work function, whereas H-PSS and Nafion enhance it. According to the proposed mechanism for PFN, the direction of the dipole in the WML at the interface is determined by the size of the constituent parts and their ability to move. In the anionic polyelectrolytes studied here the charged constituent parts are the ions within the $\mathrm{SO}_{3}{ }^{-} \mathrm{H}^{+}$group. As the $\mathrm{SO}_{3}{ }^{-}$ion is covalently bound to a polymer backbone, it has more difficulty in reaching the interface than the small, ionically bound proton. Therefore mainly the proton approaches the interface, resulting in a dipole oriented towards the interface. These results therefore demonstrate that the proposed mechanism does not only apply to the work function modification by PFN, but also by polyelectrolytes like Nafion or H-PSS.

\subsection{Ion size effects in WMLs}

According to our proposed mechanism (Figure 6) the geometrical size of the charged constituents should strongly affect the size and direction of the induced interfacial dipole. To verify whether the ion size influences the magnitude of the work function modification, the work function modification by $\mathrm{X}^{+} \mathrm{PSS}^{-}$with $\mathrm{X}^{+}=\mathrm{H}^{+}, \mathrm{Li}^{+}, \mathrm{Na}^{+}$was studied on $\mathrm{Au}$. In addition the work function modification by quaternized ammonium salts was studied as well: a series of tetrabutylammonium halides, $\left(\mathrm{TBA}^{+} \mathrm{Y}^{-}\right)$with $\mathrm{Y}^{-}=\mathrm{F}^{-}, \mathrm{Cl}^{-}, \mathrm{Br}^{-}, \mathrm{I}^{-}$; and salts $\left(\mathrm{TBA}^{+} \mathrm{Z}^{-}\right)$with $\mathrm{Z}^{-}=\mathrm{BH}_{4}^{-}, \mathrm{BF}_{4}^{-}, \mathrm{PF}_{6}^{-}$. Also the size of the alkyl group (methyl, butyl, or hexyl) in the ammonium ion was varied. The work function modification of Au by deposition of thin layers of approximately $10 \mathrm{~nm}$ is shown in Figure 9 as a function of the ion radius. The ionic radii were taken from literature. ${ }^{[44-47]}$ The radius of the ion with an underlined name is connected to the horizontal axis. The general trend that is observed in all three series $\left(\mathrm{X}^{+} \mathrm{PSS}^{-}\right.$; $\mathrm{TBA}^{+} \mathrm{Y}^{-}$; $\mathrm{TBA}^{+} \mathrm{Z}^{-}$) is that an increase in ion size is accompanied by a reduction in the magnitude of the work function modification. In $\mathrm{H}^{+} \mathrm{PSS}^{-}$a work function increase is observed as the PSS $^{-}$ion has a low movability due to its large relative size. Increasing the size of the positive counterion reduces this difference and clearly results in a reduced magnitude of the work 


\section{WILEY-VCH}

function change. In the $\mathrm{TBA}^{+} \mathrm{F}^{-}$and $\mathrm{TBA}^{+} \mathrm{BH}_{4}{ }^{-}$salts, the $\mathrm{TBA}^{+}$ion has the lowest movability compared to the counterions due to the four relatively large butyl chains. Consequently a work function reduction is expected and also observed in Figure 9. Increasing the size of the counterions again reduces the difference in movability. The experiment is in agreement with this as the magnitude of the work function reduction is reduced. That not only the ion size is important regarding work function modification is illustrated by the stronger reduction in case the larger $\mathrm{BH}_{4}^{-}$ion is used opposed to $\mathrm{F}^{-}, \mathrm{Cl}^{-}$, or $\mathrm{Br}^{-}$. Clustering and ordering of the salt molecules, which can strongly vary between the different series, is likely to play a large role as well. This is in line with earlier observations by Yang et al., ${ }^{[48]}$ where polyelectrolytes with larger ions used as interlayer were found to result in better device performance. Furthermore, it should be noted that in devices, the performance is not only determined by the work function change due to a WML, but also by additional effects of the WML on electrical transport and injection. A change in magnitude of the ammonium constituent itself leads to an oppositely expected effect: c.f. $\mathrm{TMA}^{+} \mathrm{I}^{-}$vs. $\mathrm{TBA}^{+} \mathrm{I}^{-}$and $\mathrm{TBA}^{+} \mathrm{Br}^{-}$vs. $\mathrm{THA}^{+} \mathrm{Br}^{-}$(see Figure 2i-k for the structural formulae). This may tentatively be explained by a change in salt density at the interface or the ability to block the counter ions from reaching the surface. Regarding the sign of the work function modification by the ammonium series, it is found to be opposite to the PSS ${ }^{-}$series. This corresponds to a dipole orientation such that the largest ion, PSS $^{-}$and $\mathrm{TBA}^{+}$, is the furthest away from the interface and the smaller counter ion is closest.

The dashed purple line in Figure 9 illustrates the work function modification by PFN to enable comparison with the polyelectrolytes. The work function reduction by PFN is similar in case $\mathrm{TBA}^{+} \mathrm{F}^{-}$is used. If however $\mathrm{TBA}^{+} \mathrm{PF}_{6}{ }^{-}$is used, no work function reduction is observed. The latter was also observed in Ref. $\left[{ }^{13}\right]$, where the work function modification by PEIE was measured as a function of the acidity of the solution from which it was deposited. The largest work function modification was obtained from a basic solution when PEIE with neutral amine groups was present. When using an acidic solution, by addition of $\mathrm{H}^{+} \mathrm{PF}_{6}{ }^{-}$, part 


\section{WILEY-VCH}

of the amines is protonated and electrostatically compensated by a $\mathrm{PF}_{6}{ }^{-}$counterion. In that case the magnitude of the work function modification was observed to decrease. This is in line with our measurement of $\mathrm{TBA}^{+} \mathrm{PF}_{6}{ }^{-}$on $\mathrm{Au}$ shown in Figure 9 where no work function modification is observed.

\subsection{General considerations}

The general behavior of the interlayers described in the previous paragraphs allows a consistent description of the work function changes, but we note that these can be accompanied with effects which are specific for the materials used. For example, work function reduction by $\mathrm{PFN}$ on $\mathrm{P} 3 \mathrm{HT}$ is expected but not observed experimentally. We tentatively attribute this to material specific effects that may be superimposed on, or interfere with the interface dipole formation as discussed in this paper. It is for example known that PFN in combination with fullerenes results in doping of the fullerenes. ${ }^{[20]}$ Another example is found in a recent paper by Wetzelaer et al., ${ }^{[49]}$ where an anionic electrolyte, stearate, with a relatively large counter ion, $\mathrm{Cs}^{+}$, was shown to improve electron injection from $\mathrm{Al}$ to a comparable level as $\mathrm{Ba}$. This result may be explained by the relatively large size of the $\mathrm{Cs}^{+}$ ion which might make image charge screening of the negatively charged polymer more favorable. Alternatively, n-type doping by $\mathrm{Cs}^{+}$may explain the observed results as well in this specific case. $^{[50]}$

In actual devices, where WMLs are typically sandwiched between an organic semiconductor and a metallic electrode, the situation is more complicated than the one studied here. In lowest order, oppositely directed dipoles are expected at both interfaces of the WML. Typically, the metal-WML interface dipole will be largest and improved injection may still be expected. However, the presence of substantial space charge in the semiconductor may destabilize the interfacial dipoles. Injection of electrons (holes) and the consequent formation of negative (positive) space charge in the organic semiconductor can destabilize a dipole 


\section{WILEY-VCH}

pointed away from (towards) the semiconductor. This would be an interesting topic for further study

\section{Conclusion}

In summary we have shown that WMLs based on tertiary aliphatic amines and polyelectrolytes, e.g. sulfonic acid based polymers or aliphatic quaternized ammonium salts, show similar behavior in work function modification. Work function modification occurs only on (semi-)conducting substrates and is independent of the substrate work function in the range of 3 to $5 \mathrm{eV}$. Additionally the work function modification does not depend on the WML thickness, but does depend on the relative size of the anion and cation and their respective ability to move.

The data supports a single mechanism for both work function reduction and enhancement by prompted formation of an interfacial dipole. This process is driven by the attraction of the positive and negative part of the dipole towards the interface by the image charge interaction in case the substrate is a (semi-)conductor. The geometrical size and movability of the positive and negative parts of the dipole determine which (partial) charge can get closest to the substrate, resulting in a net dipole orientation away from or towards the interface which modifies the work function. The large data set proves that the general work function modification by the WMLs studied here cannot be described by any doping mechanism, charge transfer, or spontaneous dipole orientation in the solid film after deposition. Although other mechanisms are not excluded for specific interlayer-substrate combinations, the prompted dipole formation mechanism is found to be universal for a large variety of interlayer materials deposited on a large variety of substrates and provides a guideline for developing new materials.

\section{Experimental Section}




\section{WILEY-VCH}

Device preparation: Devices that were measured consisted of a substrate covered with an electrode as shown schematically in Figure 1. The conductor was either sputtered, e.g. indium tin oxide (ITO), vacuum evaporated ( $\mathrm{Au}, \mathrm{Ag}, \mathrm{Al}, \mathrm{Ca})$ or spin coated (poly(3,4ethylenedioxythiophene)-poly(styrenesulfonate) (PEDOT:PSS, Clevios P VP AI 4083) from Heraeus). Deposition of $\mathrm{V}_{2} \mathrm{O}_{5}$ was performed similarly as described in Ref. [27]. Approximately $10 \mathrm{~nm}$ thick organic semiconductor or insulator films were deposited on top by spin coating from solution: [6,6]-phenyl-C 70 -butyric acid methyl ester $\left(\mathrm{PC}_{70} \mathrm{BM}\right.$, Solenne $\mathrm{BV}$ ), poly(3-hexylthiophene) (P3HT, Aldrich, $M_{\mathrm{n}}=54000-75000 \mathrm{~g} \mathrm{~mol}^{-1}$ ) and a blend of PC70BM and poly[2,6-(4,4-bis-(2-ethylhexyl)-4H-cyclopenta[2,1-b;3,4-b0]-dithiophene)-alt4,7-(2,1,3-benzothiadiazole)] (PCPDTBT, $M_{\mathrm{w}}=27700 \mathrm{~g} \mathrm{~mol}^{-1}$ ) from $o$-DCB, poly[\{2,5bis(2-hexyldecyl)-2,3,5,6-tetrahydro-3,6-dioxopyrrolo[3,4-c]pyrrole-1,4-diyl $\}$-alt- $\{[2,2$ '-(1,4phenylene)bisthiophene]-5,5'-diyl $\}$ ] (PDPPTPT, $M_{\mathrm{w}}=143000 \mathrm{~g} \mathrm{~mol}^{-1}$ ) from chloroform, a 1:1 weight\% blend of PDPPTPT and PC70BM from chloroform, poly[(9,9-dioctylfluorenyl2,7-diyl) (PFO), and a poly(p-phenylene vinylene) copolymer (Superyellow PPV, Merck, catalogue number PDY-132) from toluene, polystyrene (PS, Aldrich, $M_{\mathrm{w}}=35000 \mathrm{~g} \mathrm{~mol}^{-1}$ ), and poly(methylmethacrylate) (PMMA, Aldrich, $M_{\mathrm{w}}=10^{5} \mathrm{~g} \mathrm{~mol}^{-1}$ ) from toluene. On top of these conductors, organic semiconductors and insulators, a $\sim 10 \mathrm{~nm}$ thick film of poly[(9,9bis(3'-(N,N-dimethylamino)propyl)-2,7-fluorene)-alt-2,7-(9,9-dioctylfluorene)] (PFN) was deposited from either $2 \mathrm{mg} \mathrm{ml}^{-1}$ methanol $(\mathrm{MeOH})+$ acetic acid $(\mathrm{AcOH})$ (200:1 volume ratio) or $2 \mathrm{mg} \mathrm{ml}^{-1}$ toluene. Synthesis of PFN is described elsewhere. ${ }^{[1]}$ Alternatively, poly(4styrenesulfonic acid) (H-PSS, Aldrich, $M_{\mathrm{w}}=75000 \mathrm{~g} \mathrm{~mol}^{-1}$ ), poly(lithium 4-styrenesulfonate) (Li-PSS, Aldrich, $M_{\mathrm{w}}=75000 \mathrm{~g} \mathrm{~mol}^{-1}$ ) and poly(sodium 4-styrenesulfonate) (Na-PSS, Aldrich, $\left.M_{\mathrm{w}}=70000 \mathrm{~g} \mathrm{~mol}^{-1}\right)$ dissolved in ultrapure water $\left(4 \mathrm{mg} \mathrm{ml}^{-1}\right)$; tetrabutylammonium (TBA, Aldrich) tetramethylammonium (TMA, Aldrich), and tetrahexylammonium (THA, Aldrich) salts dissolved in tetrahydrofuran $\left(4 \mathrm{mg} \mathrm{ml}^{-1}\right)$, or Nafion (Aldrich, perfluorinated ion exchange resin $5 \mathrm{wt} \%$ in mixture of lower aliphatic and $\mathrm{H}_{2} \mathrm{O}$, including $45 \%$ water) diluted in 


\section{WILEY-VCH}

ethanol with a 1:200 volume ratio were deposited on top of the mentioned conductors, organic semiconductors, and insulators. Structural formulae of the above-mentioned materials are shown in Figure 2. The thickness of the layers ranged between 5 and $10 \mathrm{~nm}$. The thicknesses of the different layers were measured by a Veeco Dektak profilometer. Device preparation was performed in a $\mathrm{N}_{2}$ filled glovebox in case air-sensitive materials like $\mathrm{Ca}, \mathrm{Al}$ or $\mathrm{Ag}$ were used.

Device characterization: SKPM images were recorded in a glove box under $\mathrm{N}_{2}$ atmosphere $\left(\left[\mathrm{O}_{2}\right]<1 \mathrm{ppm}\right.$ and $\left.\left[\mathrm{H}_{2} \mathrm{O}\right]<1 \mathrm{ppm}\right)$ with a Veeco Instruments MultiMode AFM with Nanoscope IV controller, operating in lift mode with a lift height of $50 \mathrm{~nm}$. Au-coated silicon tips from MikroMasch were employed. During surface potential measurements the sample was grounded. An Au substrate was used as reference to monitor any tip wear after multiple measurements. The measurements were carried out at room temperature. As SKPM is based on nullification of the electric field between tip and sample, no driving force for ion displacement during characterization exists. The discussed ion work function shifts and ion displacements therefore have taken place upon or immediately after layer deposition and not during SKPM probing.

\section{Supporting Information}

Supporting Information is available from the Wiley Online Library or from the author.

\section{Acknowledgements}

We thank Koen Hendriks for the synthesis of PFN. SvR and MK acknowledge financial support from the Dutch program NanoNextNL. This work forms further part of the Joint Solar Programme (JSP) and of the Stichting voor Fundamenteel Onderzoek der Materie (FOM), which is supported financially by Nederlandse Organisatie voor Wetenschappelijk Onderzoek (NWO). This work is co-financed by Hyet Solar. The research leading to these results has 


\section{WILEY-VCH}

received funding from the Ministry of Education, Culture and Science (Gravity program 024.001.035) and is part of the Solliance Organic Photovoltaics Programme.

Received: ((will be filled in by the editorial staff))

Revised: ((will be filled in by the editorial staff)) Published online: ((will be filled in by the editorial staff))

[1] F. Huang, H. B. Wu, D. Wang, W. Yang, Y. Cao, Chem. Mater. 2004, 16, 708.

[2] A. K. K. Kyaw, D. H. Wang, V. Gupta, J. Zhang, S. Chand, G. C. Bazan, A. J. Heeger, Adv. Mater. 2013, 25, 2397.

[3] C. M. Zhong, C. H. Duan, F. Huang, H. B. Wu, Y. Cao, Chem. Mater. 2011, 23, 326.

[4] L. F. Lan, J. B. Peng, M. L. Sun, J. L. Zhou, J. H. Zou, J. Wang, Y. Cao, Org. Electron. 2009, 10, 346.

[5] C. V. Hoven, R. Q. Yang, A. Garcia, V. Crockett, A. J. Heeger, G. C. Bazan, T. Q. Nguyen, Proc. Natl. Acad. Sci. U S A 2008, 105, 12730.

[6] J. H. Seo, T. Q. Nguyen, J. Am. Chem. Soc. 2008, 130, 10042.

[7] J. Lee, H. Kang, J. Kong, K. Lee, Adv. Energy Mater. 2014, 4, 1301226.

[8] S. Braun, W. R. Salaneck, M. Fahlman, Adv. Mater. 2009, 21, 1450.

[9] H. Vazquez, F. Flores, A. Kahn, Org. Electron. 2007, 8, 241.

[10] A. Kahn, N. Koch, W. Y. Gao, J. Polym. Sci. Part B Polym. Phys. 2003, 41, 2529.

[11] L. Lindell, M. P. de Jong, W. Osikowicz, R. Lazzaroni, M. Berggren, W. R. Salaneck, X. Crispin, J. Chem. Phys. 2005, 122, 84712.

[12] D. E. Gardin, G. A. Somorjai, J. Phys. Chem. 1992, 96, 9424.

[13] Y. H. Zhou, C. Fuentes-Hernandez, J. Shim, J. Meyer, A. J. Giordano, H. Li, P. Winget, T. Papadopoulos, H. Cheun, J. Kim, M. Fenoll, A. Dindar, W. Haske, E. Najafabadi, T. M. Khan, H. Sojoudi, S. Barlow, S. Graham, J. L. Bredas, S. R. Marder, A. Kahn, B. Kippelen, Science 2012, 336, 327.

[14] F. Huang, H. B. Wu, Y. Cao, Chem. Soc. Rev. 2010, 39, 2500.

[15] R. D. Xia, D. S. Leem, T. Kirchartz, S. Spencer, C. Murphy, Z. C. He, H. B. Wu, S. J. Su, Y. Cao, J. S. Kim, J. C. deMello, D. D. C. Bradley, J. Nelson, Adv. Energy Mater. 2013, 3, 718.

[16] S. Liu, K. Zhang, J. Lu, J. Zhang, H.-L. Yip, F. Huang, Y. Cao, J. Am. Chem. Soc. 2013, $135,15326$.

[17] Y.-H. Kim, T.-H. Han, H. Cho, S.-Y. Min, C.-L. Lee, T.-W. Lee, Adv. Funct. Mater. 2014, 24, 3808.

[18] F. Lui, Z. A. Page, V. V. Duzhko, T. P. Russell, T. Emrick, Adv. Mater. 2013, 25, 6868.

[19] B. H. Lee, I. H. Jung, H. Y. Woo, H.-K. Shim, G. Kim, K. Lee, Adv. Funct. Mater. 2014, $24,1100$.

[20] C.-Z. Li, C.-C. Chueh, F. Ding, H.-L. Yip, P.-W. Liang, X. Li, A. K. Y. Jen, Adv. Mater. 2013, 25, 4425 .

[21] T. W. Lee, O. Kwon, M. G. Kim, S. H. Park, J. Chung, S. Y. Kim, Y. Chung, J. Y. Park, E. Han, D. H. Huh, J. J. Park, L. Pu, Appl. Phys. Lett. 2005, 87, 231106.

[22] T. W. Lee, Y. Chung, O. Kwon, J. J. Park, Adv. Funct. Mater. 2007, 17, 390.

[23] M. Manceau, S. Berson, Nanotechnology 2014, 25, 014018.

[24] G. Greczynski, T. Kugler, M. Keil, W. Osikowicz, M. Fahlman, W. R. Salaneck, J. Electron. Spectrosc. Relat. Phenom. 2001, 121, 1. 


\section{WILEY-VCH}

[25] A. M. Nardes, M. Kemerink, M. M. de Kok, E. Vinken, K. Maturova, R. A. J. Janssen, Org. Electron. 2008, 9, 727.

[26] J. Meyer, K. Zilberberg, T. Riedl, A. Kahn, J. Appl. Phys. 2011, 110.

[27] K. Zilberberg, S. Trost, H. Schmidt, T. Riedl, Adv. Energy Mater. 2011, 1, 377.

[28] J. Lim, M. Park, W. K. Bae, D. Lee, S. Lee, C. Lee, K. Char, ACS Nano 2013, 7, 9019.

[29] Q. B. Pei, G. Yu, C. Zhang, Y. Yang, A. J. Heeger, Science 1995, 269, 1086.

[30] S. van Reenen, P. Matyba, A. Dzwilewski, R. A. J. Janssen, L. Edman, M. Kemerink, J. Am. Chem. Soc. 2010, 132, 13776.

[31] M. Lenes, G. Garcia-Belmonte, D. Tordera, A. Pertegas, J. Bisquert, H. J. Bolink, Adv. Funct. Mater. 2011, 21, 1581.

[32] K. Abbas, D. Marji, Z. Naturforsch. A 2005, 60, 667.

[33] P. C. Rusu, G. Giovannetti, C. Weijtens, R. Coehoorn, G. Brocks, Phys. Rev. B 2010, 81, 125403.

[34] J. D. Jackson, Classical Electrodynamics, John Wiley \& Sons, New York 1962.

[35] H. Ishii, K. Sugiyama, E. Ito, K. Seki, Adv. Mater. 1999, 11, 972.

[36] W. C. Meixner, P. R. Antoniewicz, Phys. Rev. B 1976, 13, 3276.

[37] P. R. Hilton, D. W. Oxtoby, J. Chem. Phys. 1980, 72, 6346.

[38] B. L. Maschhoff, J. P. Cowin, J. Chem. Phys. 1994, 101, 8138.

[39] P. M. Dewick, Essentials of Organic Chemistry, John Wiley \& Sons, West Sussex 2006.

[40] R. C. G. Naber, J. Massolt, M. Spijkman, K. Asadi, P. W. M. Blom, D. M. de Leeuw, Appl. Phys. Lett. 2007, 90, 113509.

[41] J. J. Brondijk, K. Asadi, P. W. M. Blom, D. M. de Leeuw, J. Polym. Sci. Part B Polym. Phys. 2012, 50, 47.

[42] P. Wurfel, I. P. Batra, J. T. Jacobs, Phys. Rev. Lett. 1973, 30, 1218.

[43] P. Wurfel, I. P. Batra, Phys. Rev. B 1973, 8, 5126.

[44] A. L. Rohl, D. M. P. Mingos, Dalton Trans. 1992, 3541.

[45] Y. Nakamori, K. Miwa, A. Ninomiya, H. W. Li, N. Ohba, S. I. Towata, A. Zuttel, S. I. Orimo, Phys. Rev. B 2006, 74, 045126.

[46] J. C. Slater, J. Chem. Phys. 1964, 41, 3199.

[47] J. S. Huang, B. G. Sumpter, V. Meunier, Chem. Eur. J. 2008, 14, 6614.

[48] R. Q. Yang, H. B. Wu, Y. Cao, G. C. Bazan, J. Am. Chem. Soc. 2006, 128, 14422.

[49] G. A. H. Wetzelaer, A. Najafi, R. J. P. Kist, M. Kuik, P. W. M. Blom, Appl. Phys. Lett. 2013, 102, 053301.

[50] H. H. P. Gommans, M. Kemerink, G. G. Andersson, R. M. T. Pijper, Phys. Rev. B 2004, 69, 155216. 


\section{WILEY-VCH}

(a)

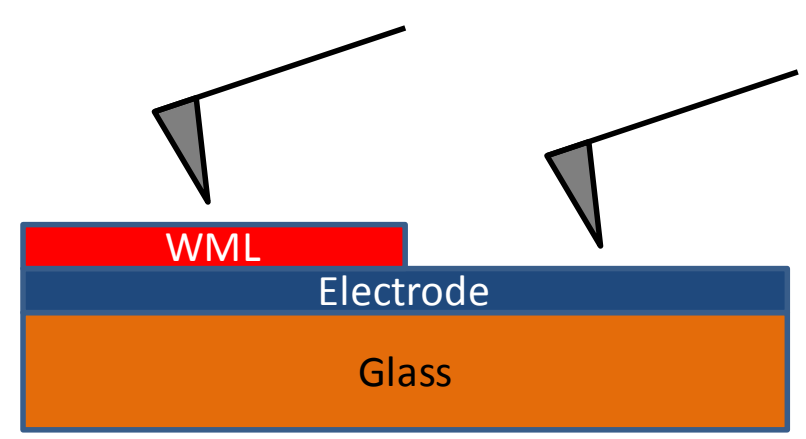

(b)

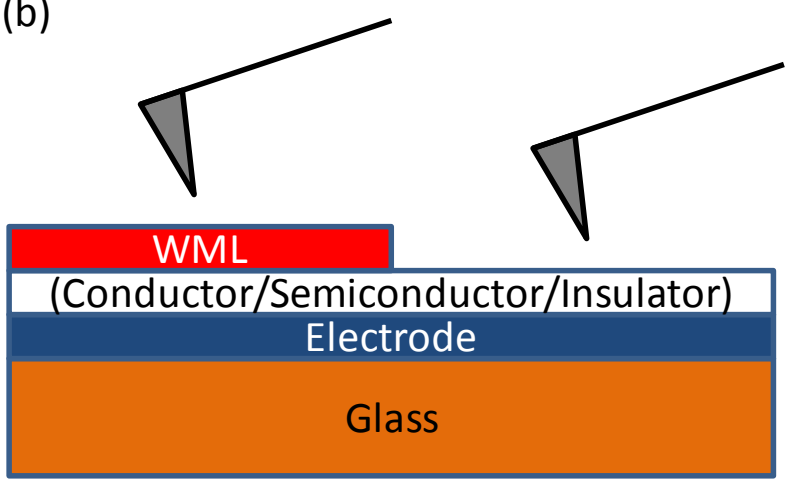

Figure 1. Schematic of the general device layout. SKPM measurements were performed on (a) metal electrodes with and without a work function modification layer (WML) on top and on (b) metal electrodes covered with a $\sim 10 \mathrm{~nm}$ layer of conductor/semiconductor/insulator with and without a work function modification layer on top. 


\section{WILEY-VCH}

(a)

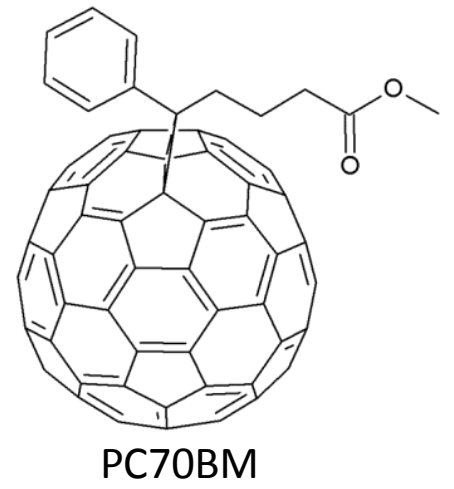

(d)

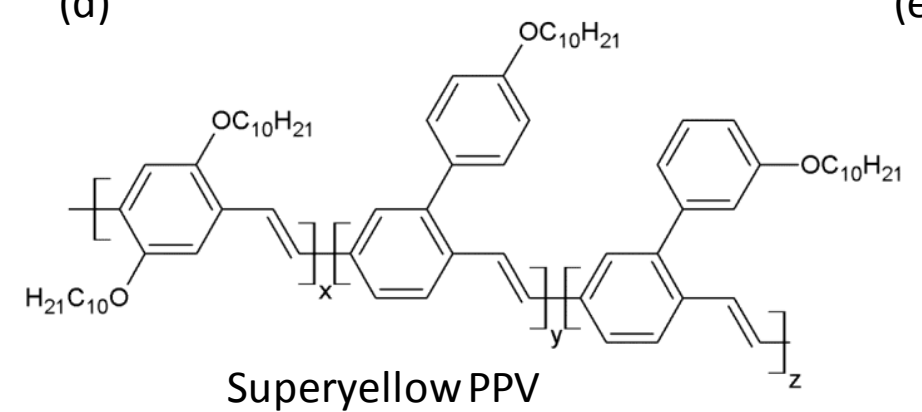

(f)

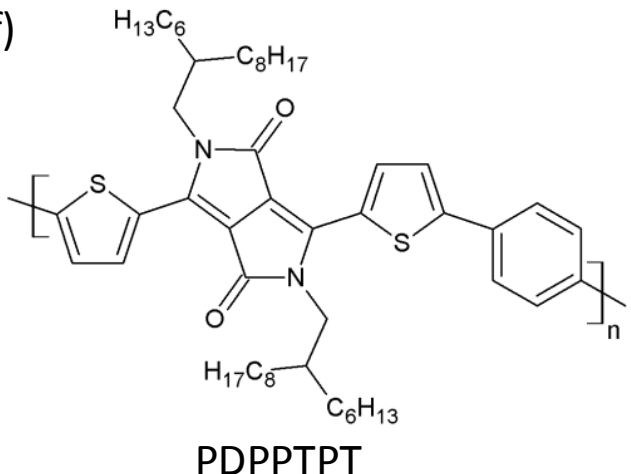

(g)

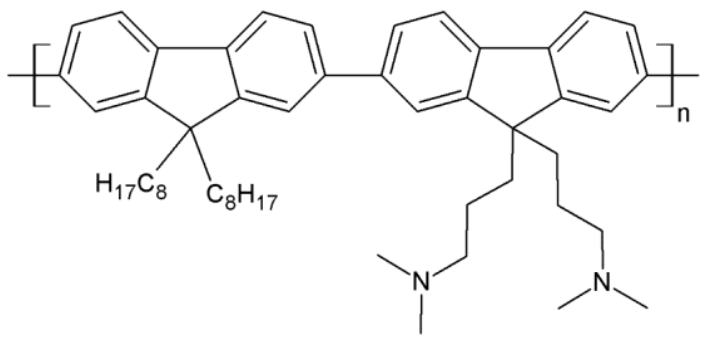

PFN (b)

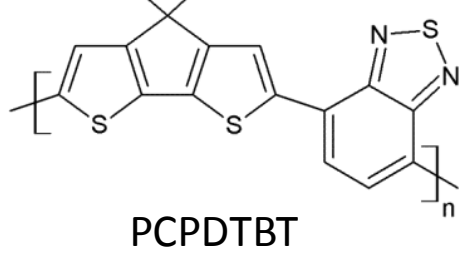

(e) (c)

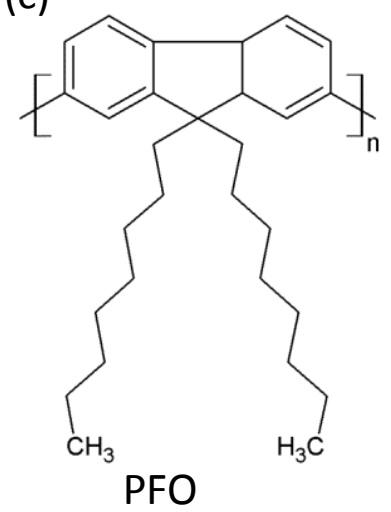

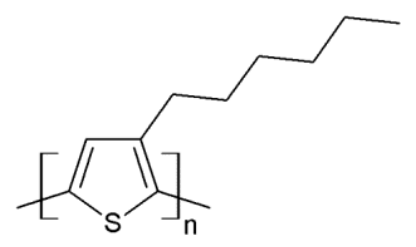

P3HT (h)

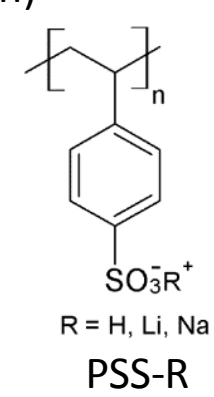

(i)

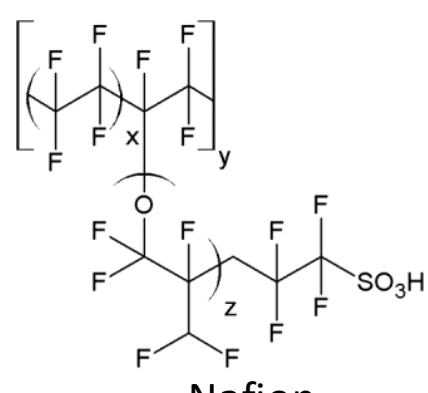

Nafion

(j)

(k)

(l)

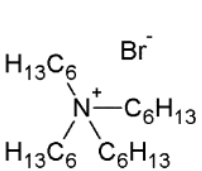

$$
\begin{aligned}
& \begin{array}{c}
\mathrm{H}_{9} \mathrm{C}_{4} \times \\
\mathrm{N}^{\mathrm{N}} \times \mathrm{C}_{4} \mathrm{H}_{9} \\
\mathrm{H}_{9} \mathrm{C}_{4} \mathrm{C}_{4} \mathrm{C}_{9} \\
\mathrm{X}=\mathrm{F}, \mathrm{Cl}, \mathrm{Br}, \mathrm{I}, \\
\mathrm{BH}_{4}, \mathrm{BF}_{4}, \mathrm{PF}_{6}
\end{array}
\end{aligned}
$$

(m)

(n)

Figure 2. Structural formulae of the organic compounds.<smiles>COC(=O)C(C)(CC(C)(C)C)C(=O)OC</smiles>

PMMA<smiles>CC(C)C(C)(C)C(C)(C)C(C)(C)C</smiles>

PS 
Table 1. Work function modification by PFN.

\begin{tabular}{lcc}
\hline Work function [eV] & Pristine & With PFN \\
\hline ITO & 4.4 & 3.65 \\
$\mathrm{Au}$ & 4.6 & 4.0 \\
$\mathrm{Ag}$ & 4.3 & 4.0 \\
$\mathrm{Al}$ & 3.07 & 2.76 \\
$\mathrm{~V}_{2} \mathrm{O}_{5}$ & 5.0 & 4.2 \\
PEDOT & 4.92 & 4.48 \\
p-type Si & 4.22 & 3.5 \\
$\mathrm{Ca}$ & 2.85 & 1.85 \\
\hline ITO-PC70BM:PCPDTBT & 4.5 & 4.04 \\
ITO-PPV & 4.55 & 4.21 \\
ITO-P3HT & 4.24 & 4.24 \\
Au-PFO & 4.42 & 4.03 \\
Au-PC70BM & 4.62 & 4.02 \\
ITO-PC70BM & 4.73 & 4.19 \\
ITO-PC70BM:PDPPTPT & 4.19 & 3.98 \\
ITO-PDPPTPT & 4.30 & 3.80 \\
\hline Au-PS & 4.44 & 4.35 \\
Au-PMMA & 4.59 & 4.49 \\
\hline
\end{tabular}

Table 2. Work function modification by H-PSS.

\begin{tabular}{lcc}
\hline Work function [eV] & Pristine & With H-PSS \\
\hline ITO & 4.4 & 5.0 \\
$\mathrm{Au}$ & 4.6 & 5.49 \\
$\mathrm{Ag}$ & 4.3 & 4.7 \\
$\mathrm{Al}$ & 3.07 & 4.1 \\
\hline ITO-PPV & 4.47 & 4.64 \\
Au-PC70BM:PCPDTBT & 4.5 & 4.7 \\
\hline Au-PMMA & 4.6 & 4.6 \\
\hline
\end{tabular}

Table 3. Work function modification by Nafion.

\begin{tabular}{lcc}
\hline Work function [eV] & Pristine & With Nafion \\
\hline ITO & 4.4 & 4.95 \\
Au & 4.6 & 5.1 \\
PEDOT & 4.85 & 5.25 \\
\hline Au-PFO & 4.48 & 4.6 \\
\hline Au-PS & 4.9 & 4.85 \\
Au-PMMA & 4.7 & 4.8 \\
\hline
\end{tabular}




\section{WILEY-VCH}

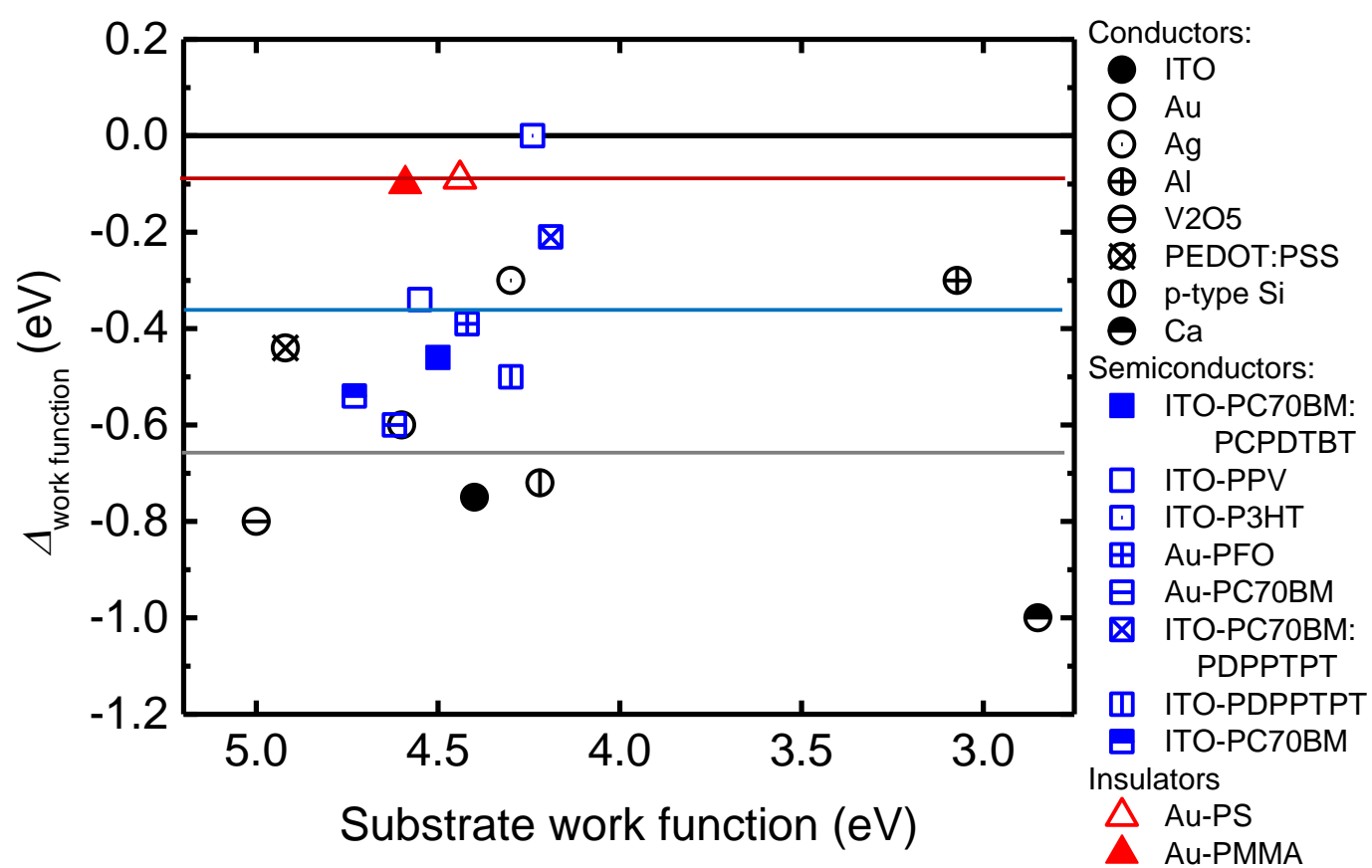

Figure 3. Work function as measured by SKPM for substrates covered by $\sim 10 \mathrm{~nm}$ PFN.

Substrates are categorized in clean conductors (black circles) and metals coated with a $5-10$ $\mathrm{nm}$ thick film of semiconductor (blue squares) or insulator (red triangles). The symbol filling indicates the specific substrate material as shown in the legend. The horizontal lines indicate the average change in work function for each substrate category. The data is also presented in Table 1. 
WILEY-VCH

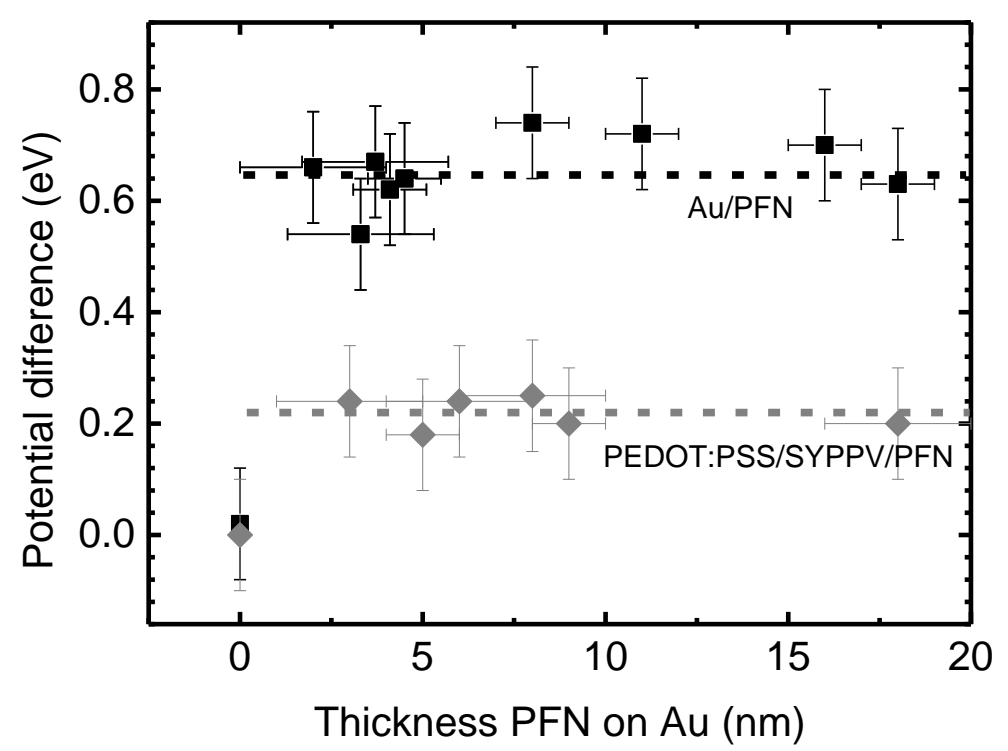

Figure 4. Potential difference as determined by SKPM between a Au tip and Au covered by PFN (black symbols) and between a Au tip and SY-PPV on PEDOT:PSS covered by PFN (gray symbols). The dashed lines are to guide the eye. 


\section{WILEY-VCH}

(a)
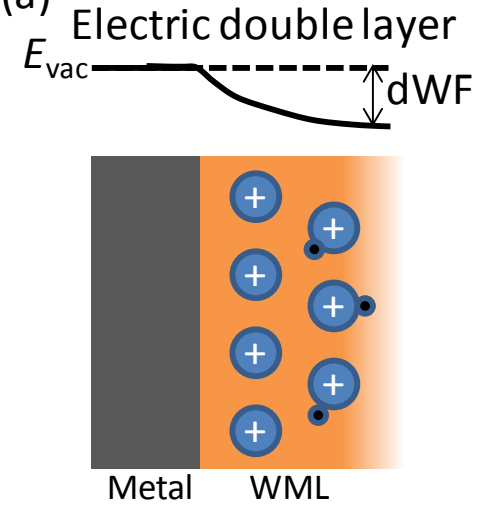

(c) Spontaneous
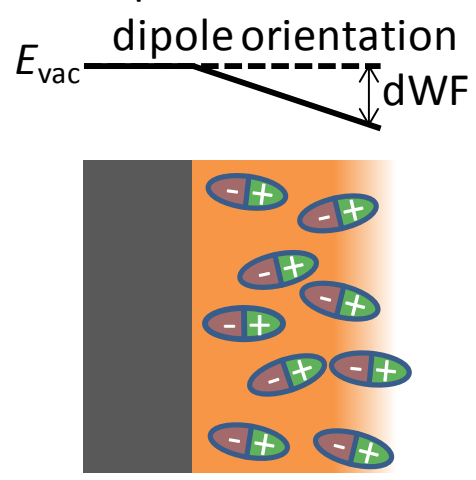

\section{Molecule}

† Oxidized molecule (b)
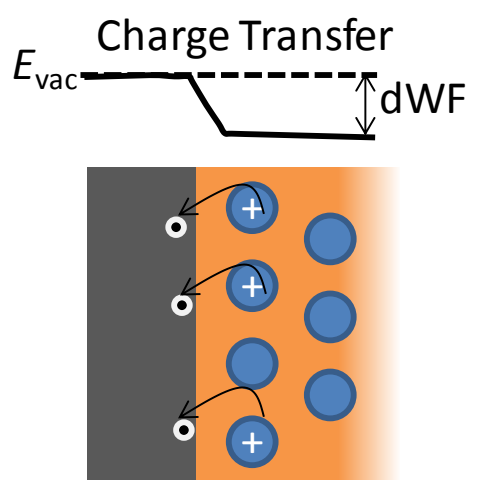

(d) Spontaneous interfacial

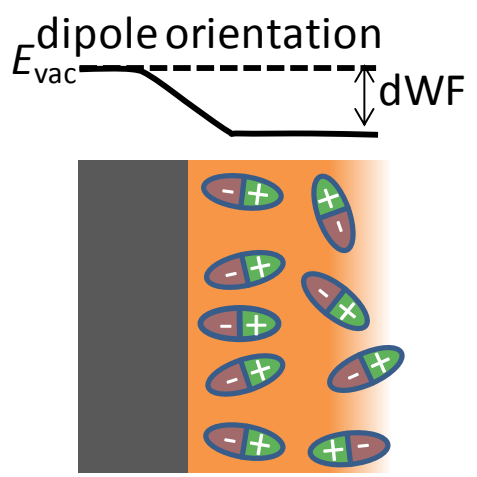

- Electron $\rightarrow$ Dipole

Figure 5. Schematics of possible mechanisms to explain the work function modification by WMLs: (a) electric double layer formation, (b) charge transfer, (c) spontaneous dipole orientation in the bulk and (d) spontaneous dipole orientation at the interface. The corresponding vacuum levels are shown on top to indicate the spatial potential drop. A legend is shown below. 
WILEY-VCH

(a)

$$
E_{\mathrm{vac}}
$$

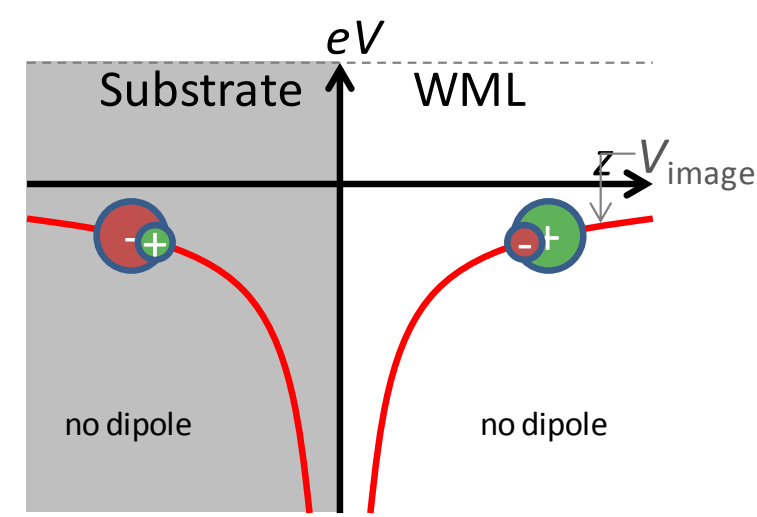

(b)

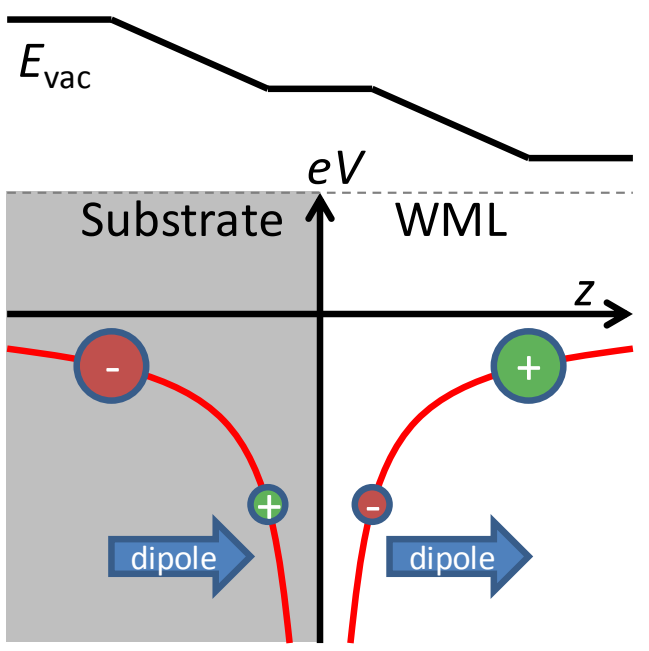

Figure 6. Schematics of the formation of an interfacial dipole between a WML and the substrate by image charge attraction. The red line indicates the image potential (a) In case the charges in the WML have similar ability to move no dipole is formed. (b) In case the charges in the WML differ in movability, a dipole is formed as the more mobile charge has a smaller approach distance towards the interface. The image charges form a dipole as well, equal in size and direction as the real dipole. The resulting work function modification is shown by the vacuum level indicated as black line. 


\section{WILEY-VCH}

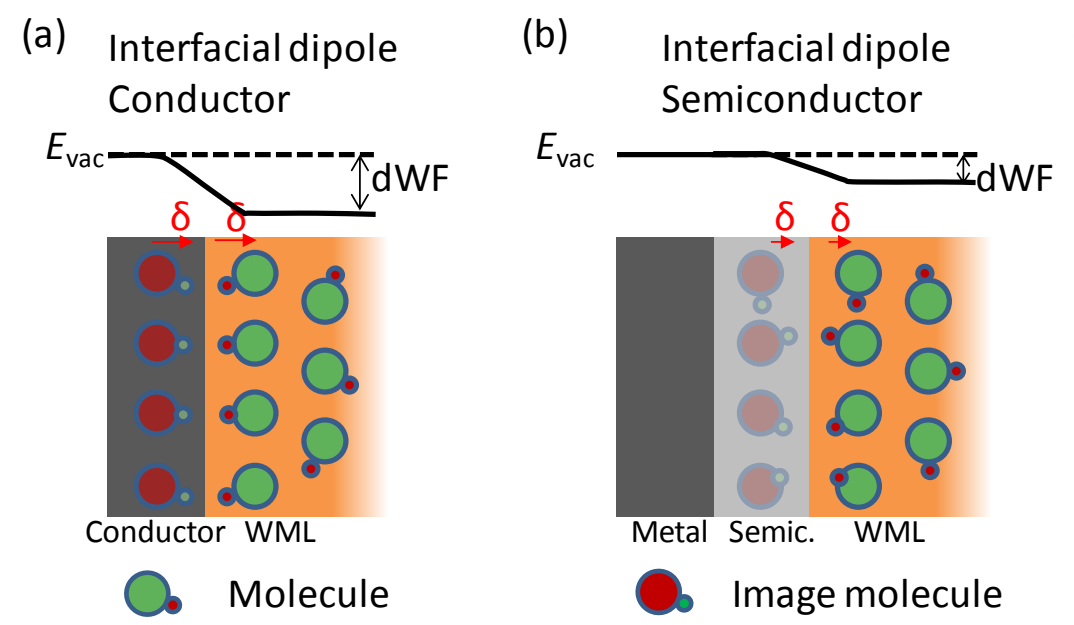

(c) Interfacial dipole

Insulator
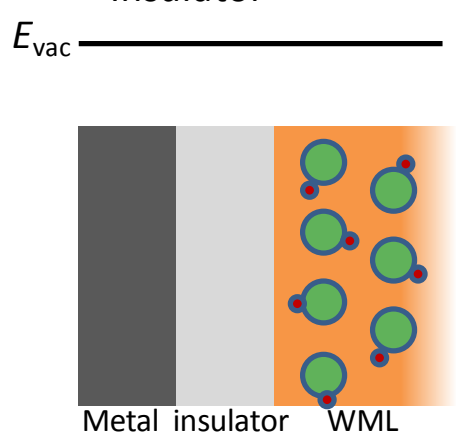

Figure 7. Schematics of the proposed work function modification mechanism. Shown is the formation of an interfacial dipole $(\delta$ ) between a WML and (a) a conductor, (b) a semiconductor and (c) an insulator. The full, partial and absent stabilization by (image) charges in the substrate is also shown. The corresponding vacuum levels are shown on top to indicate the spatial potential drop. A legend is shown below. 


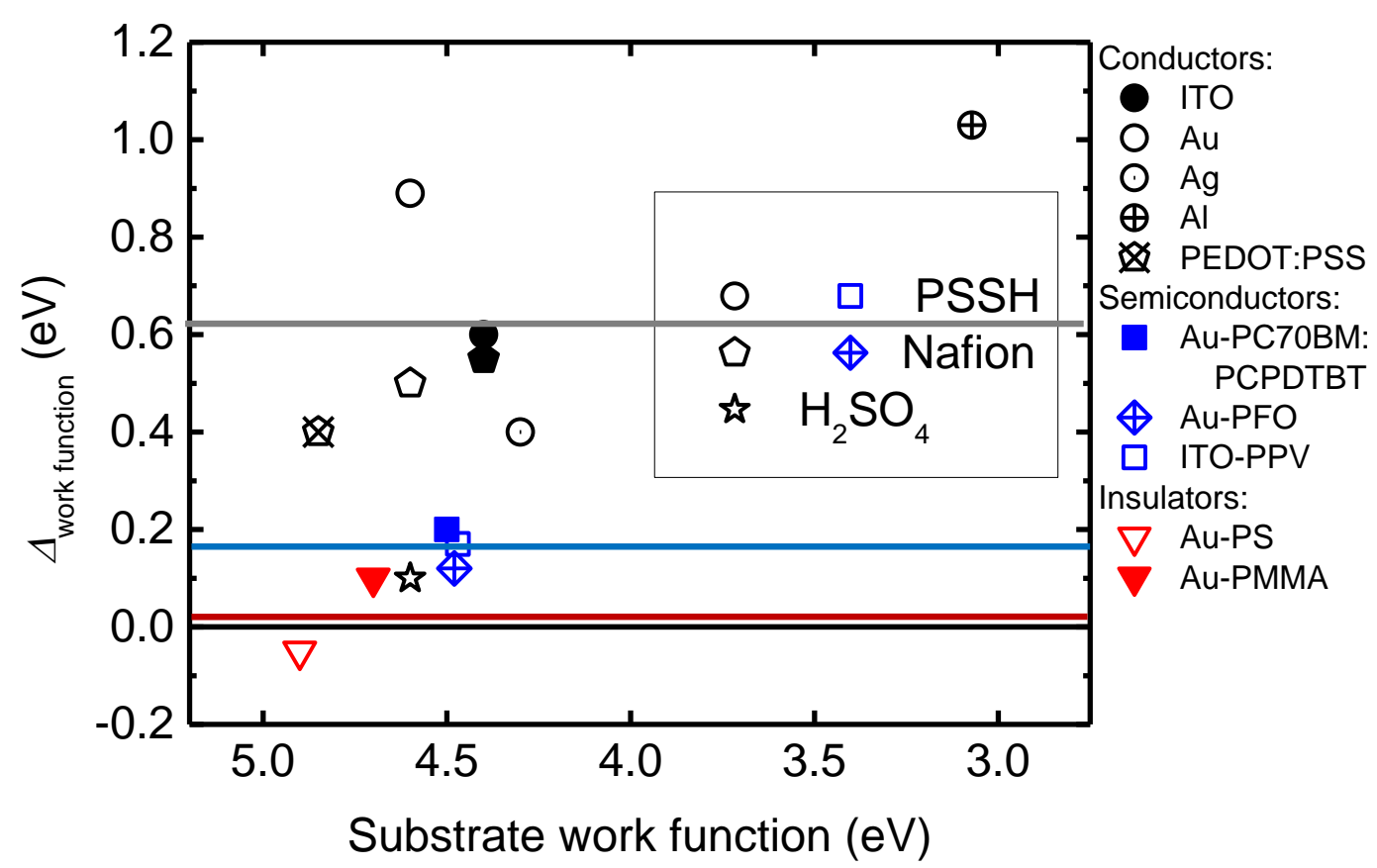

Figure 8. Work function as measured by SKPM for substrates covered by $\sim 10 \mathrm{~nm}$ H-PSS or Nafion. Substrates are categorized in clean conductors (black circles or pentagons for H-PSS and Nafion, respectively) and metals coated with a 5-10 nm thick film of semiconductor (blue squares or diamonds for H-PSS and Nafion, respectively) or insulator (red triangles for Nafion). The symbol filling indicates the specific substrate material as shown in the legend. The horizontal lines indicate the average change in work function for each substrate category. The data is also presented in Table 2 and 3. 
WILEY-VCH

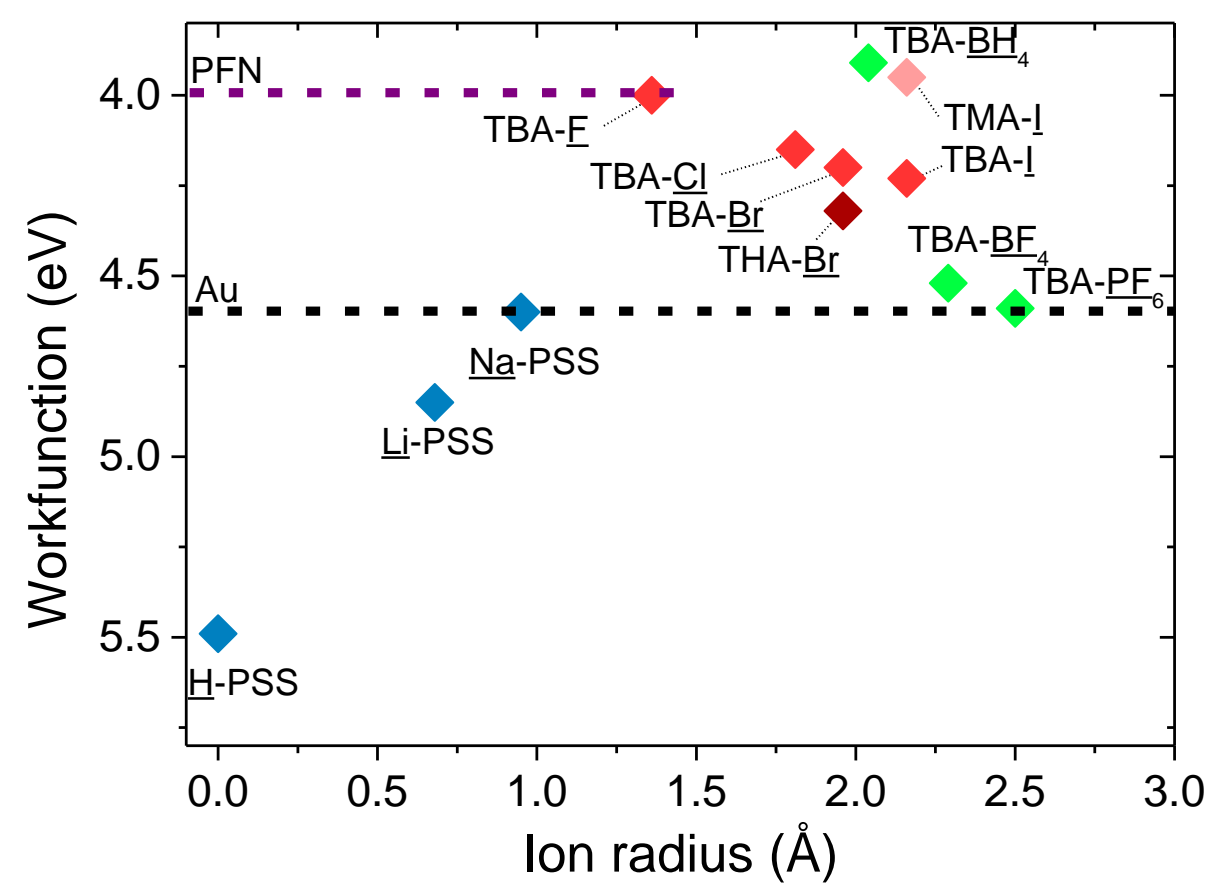

Figure 9. Work function of an Au substrate covered by a thin layer (5-10 nm) of salt as measured by SKPM. The labels show the type of ion and the ion radius of the underlined ion corresponds to the horizontal axis. The dashed lines indicate the work function of a clean $\mathrm{Au}$ substrate and an Au substrate covered with PFN. 


\section{WILEY-VCH}

Work function modification by interlayers based on tertiary aliphatic amines and polyelectrolytes is found to follow a general mechanism. When deposited on conductors and semiconductors an interfacial dipole forms, induced by image charges in the substrate. The size and movability of the charged constituents forming the dipole determines the dipole strength and direction.

\section{Keyword}

Stephan van Reenen, Sandra Kouijzer, René A. J. Janssen, Martijn M. Wienk, and Martijn Kemerink*

Origin of work function modification by ionic and amine-based interface layers

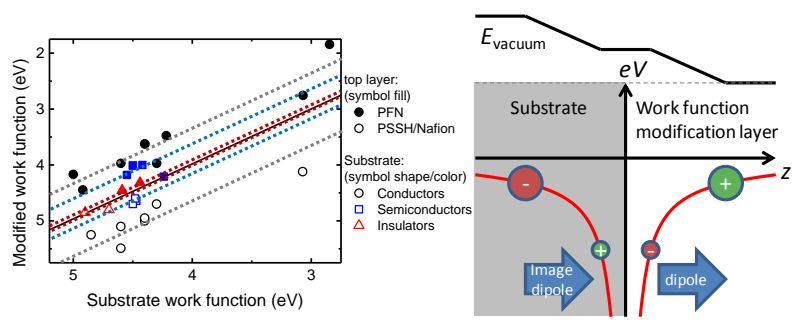




\section{WILEY-VCH}

Copyright WILEY-VCH Verlag GmbH \& Co. KGaA, 69469 Weinheim, Germany, 2013.

\section{Supporting Information}

\section{Origin of work function modification by ionic and amine-based interface layers}

Stephan van Reenen, Sandra Kouijzer, René A. J. Janssen, Martijn M. Wienk, and Martijn Kemerink*

To verify whether PFN indeed improves electron collection by Al similarly as LiF, solar cells with the stack ITO|PEDOT:PSS|PCDTBT:PC70BM|WML|Al were prepared in which WML was either PFN, LiF or nothing. Current-voltage characteristics are shown in Figure S1. In Table S1 a list is given of the corresponding short-circuit current $\left(J_{\mathrm{sc}}\right)$, open-circuit voltage $\left(V_{\mathrm{oc}}\right)$, Fill factor $(\mathrm{FF})$, the maximum power point $(\mathrm{MPP})$, and the photon conversion efficiency (PCE).

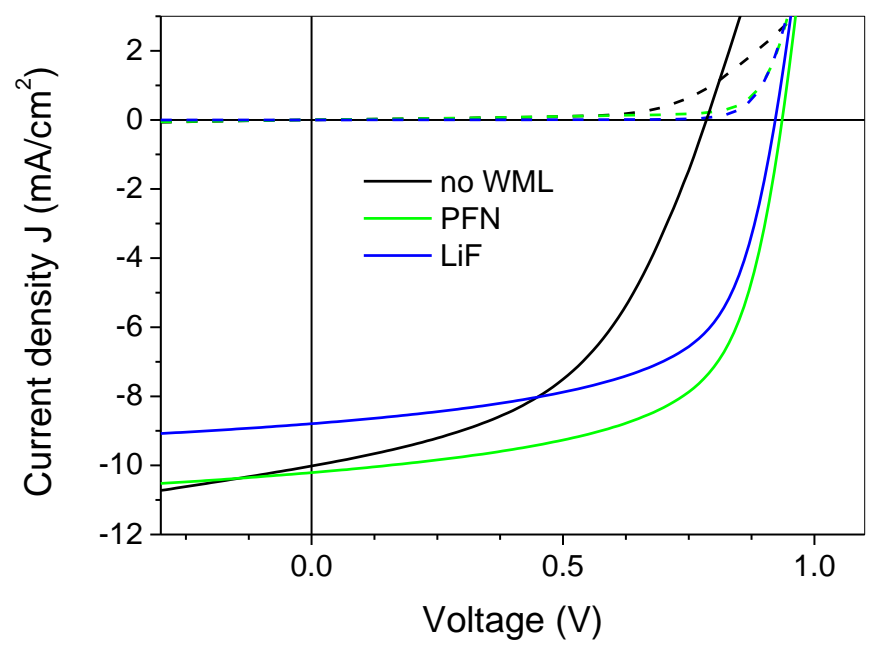

Figure S1. Current-voltage characteristics of

ITO|PEDOT:PSS|PCDTBT:PC $70 \mathrm{BM} \mid$ WML|Aluminum solar cells with varying WML as indicated in the legend.

The device without WML shows a normal short circuit current but low open circuit voltage and fill factor. With $1 \mathrm{~nm} \mathrm{LiF}$ this device shows the correct voltage, and when LiF is replaced 


\section{WILEY-VCH}

by $0.15 \mathrm{mg} \mathrm{ml}^{-1} \mathrm{PFN}$ in methanol the current increases. Altogether the results indicate that the electron collecting contact is improved by insertion of PFN between the active layer and the aluminum electrode.

Table S1. Parameters extracted from Figure S1.

\begin{tabular}{|c|c|c|c|c|c|}
\hline WML & $\begin{array}{l}V_{\text {oc }} \\
{[\mathrm{V}]}\end{array}$ & FF & $\begin{array}{l}J_{\text {sc }} \\
{\left[\mathbf{m A ~} \mathbf{m}^{-2}\right]}\end{array}$ & 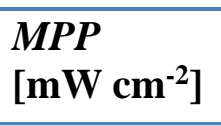 & $\begin{array}{l}P C E \\
{[\%]}\end{array}$ \\
\hline No & 0.78 & 0.48 & 10.0 & 3.78 & - \\
\hline PFN & 0.94 & 0.62 & 10.2 & 5.91 & 5.76 \\
\hline $\mathrm{LiF}$ & 0.92 & 0.61 & 8.79 & 4.93 & 4.81 \\
\hline
\end{tabular}




\section{WILEY-VCH}

Copyright WILEY-VCH Verlag GmbH \& Co. KGaA, 69469 Weinheim, Germany, 2013.

\section{Supporting Information}

\section{Origin of work function modification by ionic and amine-based interface layers}

Stephan van Reenen, Sandra Kouijzer, René A. J. Janssen, Martijn M. Wienk, and Martijn Kemerink*

To verify whether PFN indeed improves electron collection by Al similarly as LiF, solar cells with the stack ITO|PEDOT:PSS|PCDTBT:PC70BM|WML|Al were prepared in which WML was either PFN, LiF or nothing. Current-voltage characteristics are shown in Figure S1. In Table S1 a list is given of the corresponding short-circuit current $\left(J_{\mathrm{sc}}\right)$, open-circuit voltage $\left(V_{\mathrm{oc}}\right)$, Fill factor $(\mathrm{FF})$, the maximum power point $(\mathrm{MPP})$, and the photon conversion efficiency (PCE).

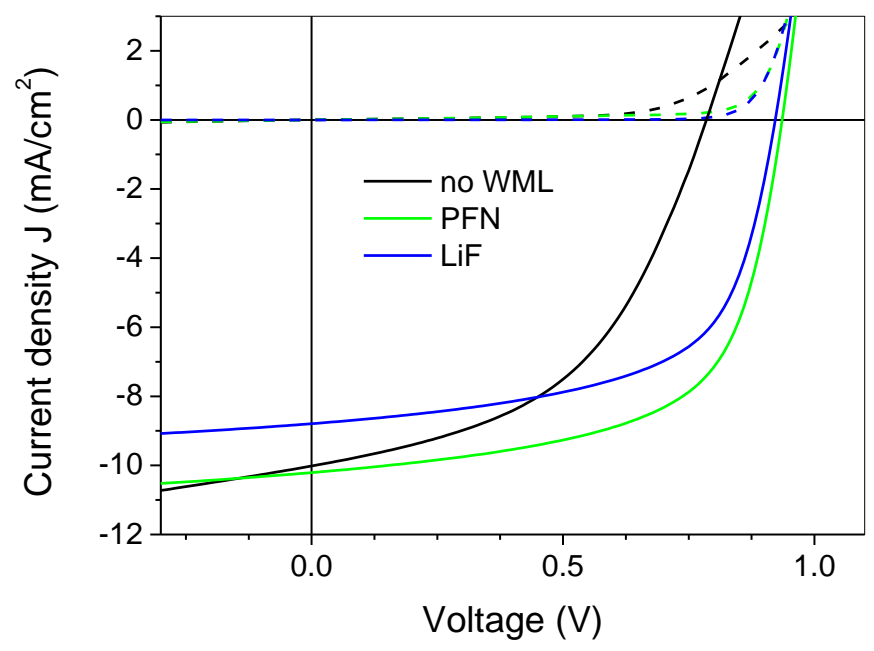

Figure S1. Current-voltage characteristics of

ITO|PEDOT:PSS|PCDTBT:PC70BM|WML|Aluminum solar cells with varying WML as indicated in the legend.

The device without WML shows a normal short circuit current but low open circuit voltage and fill factor. With $1 \mathrm{~nm} \mathrm{LiF}$ this device shows the correct voltage, and when LiF is replaced 


\section{WILEY-VCH}

by $0.15 \mathrm{mg} \mathrm{ml}^{-1} \mathrm{PFN}$ in methanol the current increases. Altogether the results indicate that the electron collecting contact is improved by insertion of PFN between the active layer and the aluminum electrode.

Table S1. Parameters extracted from Figure S1.

\begin{tabular}{|c|c|c|c|c|c|}
\hline WML & $\begin{array}{l}V_{\text {oc }} \\
{[\mathrm{V}]}\end{array}$ & FF & $\begin{array}{l}J_{\text {sc }} \\
{\left[\mathbf{m A ~} \mathbf{m}^{-2}\right]}\end{array}$ & 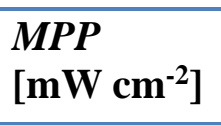 & $\begin{array}{l}P C E \\
{[\%]}\end{array}$ \\
\hline No & 0.78 & 0.48 & 10.0 & 3.78 & - \\
\hline PFN & 0.94 & 0.62 & 10.2 & 5.91 & 5.76 \\
\hline $\mathrm{LiF}$ & 0.92 & 0.61 & 8.79 & 4.93 & 4.81 \\
\hline
\end{tabular}

